\title{
Uncertainty evaluation by gamma transmission measurements and CFD model comparison in a FCC cold pilot unit
}

\author{
C.C. Dantas ${ }^{1, \star}$, A.E. Moura ${ }^{1}$, H.J.B. de Lima Filho ${ }^{2}$, S.B. Melo ${ }^{3}$, V.A. Dos Santos ${ }^{4}$, and E.A.O. Lima ${ }^{5}$ \\ 1 Department of Nuclear Energy-DEN/UFPE, Federal University of Pernambuco, Recife, Pernambuco, Brazil \\ 2 Department of Chemical Engineering-DEQ/UFPE, Federal University of Pernambuco, Recife, Pernambuco, Brazil \\ 3 Informatics Center-CIN/UFPE, Federal University of Pernambuco, Recife, Pernambuco, Brazil \\ 4 Department of Chemistry-UNICAPE, Catholic University of Pernambuco, Recife, Pernambuco, Brazil \\ ${ }^{5}$ Department of Mathematics-UPE, Universidade de Pernambuco, Recife, Pernambuco, Brazil
}

Received: 2 November 2012 / Accepted: 22 November 2012

\begin{abstract}
The solid flow in air-catalyst in circulating fluidized bed was simulated with CFD model to obtain axial and radial distribution. Therefore, project parameters were confirmed and steady state operation condition was improved. Solid holds up axial end radial profiles simulation and comparison with gamma transmission measurements are in a good agreement. The transmission signal from an ${ }^{241} \mathrm{Am}$ radioactive source was evaluated in $\mathrm{NaI}(\mathrm{Tl})$ detector coupled to multichannel analyzer. This non intrusive measuring set up is installed at riser of a cold pilot unit to determine parameters of FCC catalyst flow at several concentrations. Mass flow rate calculated by combining solid hold up and solid phase velocity measurements was compared with catalyst inlet measured at down-comer. Evaluation in each measured parameter shows that a relative combined uncertainty of $6 \%$ in a $95 \%$ interval was estimated. Uncertainty analysis took into account a significant correlation in scan riser transmission measurements. An Eulerian approach of CFD model incorporating the kinetic theory of granular flow was adopted to describe the gas-solid two-phase flows in a multizone circulating reactor. Instantaneous and local gas-particle velocity, void fraction and turbulent parameters were obtained and results are shown in $2 \mathrm{D}$ and $3 \mathrm{D}$ graphics.
\end{abstract}

Keywords: FCC fluid dynamics; gamma ray scanner; CFD simulation; uncertainty valuation; solid flow

\section{Introduction}

In the industrial tomography history, industrial process development and research work along as users of the density equation, which is a Beer-Lambert based equation. The CT - Gamma Ray Computed Tomography in process engineering actually presents a rather sophisticated technological stage. Investigations on FCC - Fluid Catalytic Cracking units and on multiphase flow in opaque reactors are increasing in continuous scientific and technological developments. Nevertheless, industrial riser tomography reveals problems that often originate from the gamma transition measurements. As it is a combination of physical measuring and mathematical models a shared approach to both should be carried out, to achieve a consistent tomography development. Following this, experimental and theoretical investigations, of the reactor fluid dynamics, were adopted. The problems in industrial gamma-ray tomography and previously in pilot and laboratory experiments can be identified at first by noise during gamma transition measurements. A complex family of secondary radiations is produced by gamma interaction

^ Correspondence: ccd@ufpe.br with absorbers. As these radiations reach the detector a disturbing noise might be induced. To understand the fluid dynamic by means of flow parameters the right direction is quantifying flow patterns and mixing of phases in the systems. That ideal flow patterns like plug flow or perfect mixing are rarely achievable, and the extent of the departure from these ideal reactor concepts depends on the scale of the reactor. The framework for doing this is provided by the interpenetrating of Eulerian model and, for lean dispersed systems, by the Lagrangian-Eulerian approach. The advances made in multiphase reaction engineering by CFD are well described and summarized [1].

The real goal of a simulation project is to run the models and try to understand the results. To make it effective, it is needed to plan out before doing the runs, since just trying different things to see what happens can be a very inefficient way of attempting to learn about your models behaviors [2]. Careful planning of how to make experiments with models will generally repay big dividends in terms of how to effectively learn about the systems and how to exercise the models further. Factorial experimental designs involve the comparison of the effects of two or more variables acting simultaneously on a common response or criterion variable [3]. Principal component analysis is 


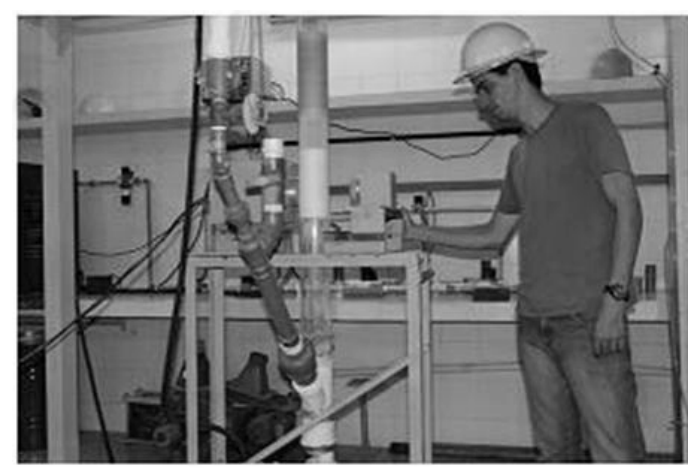

Fig. 1. Cold Pilot Unit - Instrumental set up.

appropriate when it has been measured a number of observed variables and it is desired to develop a smaller number of artificial variables that will account for most of the variance in the observed variables [4]. In this work a multivariate statistical technique was used. A composite central rotatable design DCCR was performed. In this system is required to validate fluid dynamics models by means of experimental measurements, therefore, the uncertainty evaluation, certainly, has a critical role in future development.

\section{Material and data analysis}

\subsection{Cold pilot unit}

A Cold Flow Pilot Unit (CFPU) was manufactured (Fig. 1), using similar dynamic criteria to reproduce the ascending flow of the gas solid mixture, with diluted transport of solids [2]. The CFPU had a great part of its components manufactured in transparent material to permit the visualization of important flow parameters [5]. The riser was manufactured from an acrylic tube of $0.097 \mathrm{~m}$ in inner diameter and $5.87 \mathrm{~m}$ in length. Four cyclones are responsible for the separation of the mixture cracking catalyst and compressed air at the top of the system. A return column is responsible for the transport of the catalyst to the base of the riser, also made of acrylic.

A cracking catalyst, with average diameter of $70 \mu \mathrm{m}$, with specific mass of $1500 \mathrm{~kg} / \mathrm{m}^{3}$, as well as compressed air at $103 \mathrm{kPa}$, were used as a components of the mixture through the riser. A system for the treatment and monitoring of the air quality aided in the maintenance of adequate operational conditions of the CFPU. Control strategies were used [6] with the aid of a PLC. To maintain flow conditions through the CFPU riser in stationary state its operational conditions are computer controlled.

\subsection{Gamma-ray measurements}

An arrangement to install detector and gamma source shielding on the experimental cold model riser was constructed. The ${ }^{241} \mathrm{Am}$ isotopic source of $7.4 \times 10^{-9} \mathrm{~Bq}$ intensity as well as the detector were collimated. The arrangement is mounted on a support that allows dislocating both source and detector in order to scan along the riser radius. The gamma-measurements were taken with a $2^{\prime \prime} \times$ $2^{\prime \prime} \mathrm{NaI}(\mathrm{Tl})$ detector coupled to a multichannel analyser which was provided by a Genie 2000 Canberra software for spectrum evaluation. By integrating the $0.060 \mathrm{MeV}$ photopeak of the ${ }^{241} \mathrm{Am}$ gamma-spectrum all the attenuation measurements were taken. The catalyst mass absorption coefficient, $\mu=331 \mathrm{~m}^{2} \mathrm{~kg}^{-1}$, was determined in a previous experiment. The computational control of the CFPEU operations includes monitoring graphics of the measured process variables. In order to evaluate measurement uncertainties a data sheet is generated, and then imported into the Matlab software, the monitoring graphics are then reconstructed and the required calculations are carried out. In Figure 1 a general view of the UPF is given.

\subsection{Source of errors}

The main sources of errors in tomography process are [7]:

- contribution of measurement system;

- geometric magnification factor;

- tomography reconstruction algorithms.

For the FCC to process the irradiation geometry of the riser, which is a metal cylinder, the tube wall effect is included as source of errors. The error contribution of a measurement system is given in [8], while the geometric factor is considered in [9]. The tube wall effect was evaluated by means of a mathematical model developed to simulate attenuation and estimate errors [10]. Aiming at industrial process tomography, reconstruction algorithms with reduced number of data were studied [11].

For transmission measurements using a gamma scan procedure the error contribution of the measurement system was evaluated. Due to a relatively high intensity of ${ }^{241} \mathrm{Am}$ radioactive source, a family of secondary radiations are produced by gamma interaction with absorbers. As these radiations reach the detector a disturbing noise might be induced. In addition, at a counting rate as high as $50 \mathrm{kHz}$ some spectrum perturbation should be expected. Such counting rate is associated with a high dead time that is instrumentally evaluated and corrected for the real counting time. A dead time kept under $20 \%$ is adequate taking into account the constant electronic time. The highest intensity that corresponds to $I_{0}$ was $25 \mathrm{kHz}$ in all measurements and at a dead time of $13 \%$. Due to the geometric dependence of radiation interaction, an adequate design for collimators and detector shielding should improve the gamma spectra, within practical limits. Making gamma scan measurements ends up producing a graph of parabolic shape, for intensity versus radius of tube-riser. Following the parabolic shape, nearing wall, the intensity decreases and the errors increase according to a Poisson standard deviation. In that region the tube irregularity and asymmetry normally enhance the errors due to transmission measurements. 
The irradiation geometry in the CFPU, a Plexiglas riser, does not present an influence as strong as that observed in the riser of real industrial processes. But the attenuation is relatively much higher with a ${ }^{241} \mathrm{Am}$ $0.060 \mathrm{MeV}$ gamma ray than at higher energy interval used in industrial process, therefore, errors due to irregularity and asymmetry in UPF riser were taken into account.

\section{Models and parameters}

\subsection{Simulation models}

Mathematical modeling of a gas-solid two-phase flow can be represented by two classes of models: Eulerian (continuous) and Lagrangian (discrete particles). For the present study, we adopted an approach to model the Eulerian flow with fractions diluted solids, considering that this is the most complex multiphase model available in FLUENT version 12. Eulerian models consider both phases as a continuous and fully interpenetrating. Following this approach, the equations of each phase are solved separately. To accurately describe the equations governing this type of flow the physical properties of each phase were specified.

The equations used in this study are based on the kinetic theory of Eulerian-granular model, developed in [12]. This theory has been proven and validated by many researchers: see $[13,14]$. This model allows for the presence of two different phases in one control volume of the grid by introducing the volume fraction variable. The solid phase contains spherical granular particles of the same diameter. These two phases are solved individually using the mass and momentum equations:

Mass conservation equation of gas-phase:

$$
\frac{\partial}{\partial t}\left(\varepsilon_{g} \rho_{g}\right)+\nabla \cdot\left(\varepsilon_{g} \rho_{g} v_{g}\right)=0 .
$$

Mass conservation equation of solid-phase:

$$
\frac{\partial}{\partial t}\left(\varepsilon_{s} \rho_{s}\right)+\nabla \cdot\left(\varepsilon_{s} \rho_{s} v_{s}\right)=0 .
$$

Momentum conservation equation of gas-phase:

$$
\begin{aligned}
\frac{\partial}{\partial t}\left(\varepsilon_{g} \rho_{g} v_{s}\right)+\nabla \cdot\left(\varepsilon_{g} \rho_{g} v_{g} v_{g}\right)= & -\varepsilon_{g} \nabla P+\nabla \tau_{g} \\
& +\varepsilon_{g} \rho_{g} g+\beta\left(v_{s}-v_{g}\right) .
\end{aligned}
$$

Momentum conservation equation of solid-phase:

$$
\begin{aligned}
\frac{\partial}{\partial t}\left(\varepsilon_{s} \rho_{s} v_{s}\right)+\nabla \cdot\left(\varepsilon_{s} \rho_{s} v_{s} v_{s}\right)= & -\varepsilon_{g} \nabla P+\nabla \tau_{s}-\nabla p_{s} \\
& +\varepsilon_{s} \rho_{s} g+\beta\left(v_{g}-v_{s}\right) .
\end{aligned}
$$

The equations describing the turbulence in this study model patterns, and according to [15] this is a semiempirical-two-equation model, such that one equation is for the turbulent kinetic energy (Eq. (5)), and the other one is for the rate of dissipation of turbulent kinetic energy (Eq. (6)). The constants employed in the above equations are: $C_{1 \varepsilon}=1.44 ; C_{2 \varepsilon}=1.92 ; C_{\mu}=0.09 ; \sigma_{\varepsilon}=1.0$ and $\sigma_{\mathrm{K}}=1.0$. The mixture properties are described by equations (7) and (8).

$$
\begin{aligned}
\frac{\partial}{\partial t}\left(\rho_{m} k\right)+\nabla \cdot\left(\rho_{m} v_{m} k\right)= & \nabla \cdot\left(\frac{\mu_{t, m}}{\sigma_{k}}\right)+G_{k, m}-\rho_{m} \varepsilon \\
\frac{\partial}{\partial t}\left(\rho_{m} \varepsilon\right)+\nabla \cdot\left(\rho_{m} v_{m} \varepsilon\right)= & \nabla \cdot\left(\frac{\mu_{t, m}}{\sigma_{\varepsilon}}\right) \\
& +\frac{\varepsilon}{k}\left(C_{1 g} G_{k, m}-C_{2 g} \rho_{m} \varepsilon\right) \\
\rho_{m}= & \varepsilon_{g} \rho_{g}+\varepsilon_{s} \rho_{s} \\
v_{m}= & \frac{\left(\varepsilon_{g} \rho_{g} v_{g}+\varepsilon_{s} \rho_{s} v_{s}\right)}{\left(\varepsilon_{g} \rho_{g}+\varepsilon_{s} \rho_{s}\right)}
\end{aligned}
$$

\subsection{Solid hold up}

Measurements by gamma-ray transmission for the solidphase are usually modeled by the following equation:

$$
\varepsilon_{s}=\frac{1}{\mu \rho D} \ln \left(\frac{I_{\mathrm{V}}}{I_{\mathrm{F}}}\right)
$$

where $\mu$ is the mass attenuation coefficient $\left(\mathrm{cm}^{2} \mathrm{~g}^{-1}\right), \rho$ is the catalyst density $\left(\mathrm{g} \mathrm{cm}^{-3}\right), D$ is the riser internal diameter, while $I_{\mathrm{V}}$ and $I_{\mathrm{F}}$ are the gamma intensities with empty tube and at flow conditions.

For the measurements at the diameter, ten repetitions are done to evaluate solid flow with up to four different concentrations.

Graphics of $I_{\mathrm{V}}$ and $I_{\mathrm{F}}$ at different solid concentrations, plotting also the confidence interval as a control chart were made. In total of five graphs, observing the ten repetitions by counting the dispersion around the mean value, were observed. In this way the steady state of the pilot unit could be monitored. Such an observation shows a nearly equal stability for all the five graphs. In Figure 2, the samples of the highest concentration and limits of $2 \sigma$ can be seen.

The distribution of samples follows from $I_{\mathrm{V}}$ empty tube to the lowest $I_{\mathrm{F}}$ mean value that corresponds to higher concentration which is in Figure 2. In all the samples the distribution around mean is within $2 \sigma$ limits, for the relative small size of sample $n=10$, considered. The bottom limit is the $I_{\mathrm{F}}$ value coming closer to the $I_{\mathrm{V}}$ intensity as the concentration goes down, as it can be seen in Figure 3.

The lowest catalyst hold up in Figure 3, which is presented as a star, shows that the relative intensity $I_{\mathrm{V}} / I_{\mathrm{F}}$ is close to 1 with its hold up being close to zero, so a precise determination cannot be made, only the bottom limit can be estimated. 


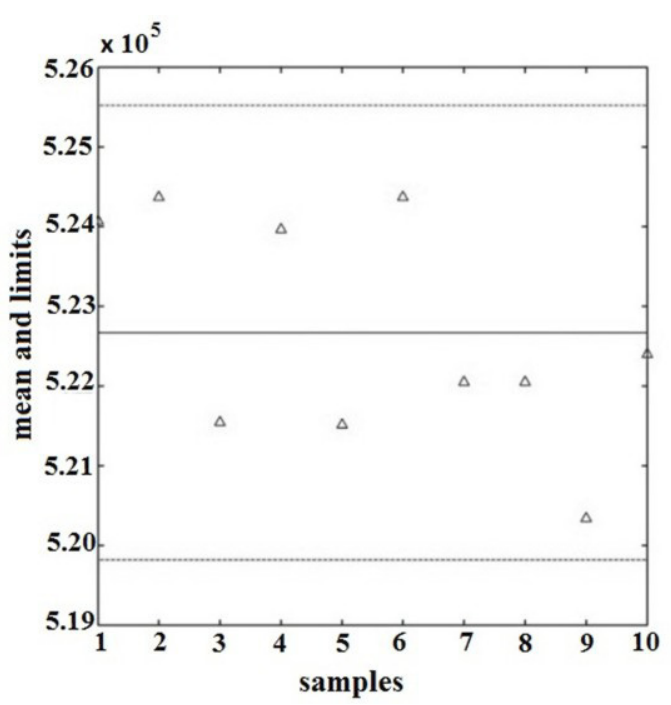

Fig. 2. Samples of the highest solid concentration around mean in a $95 \%$ interval.

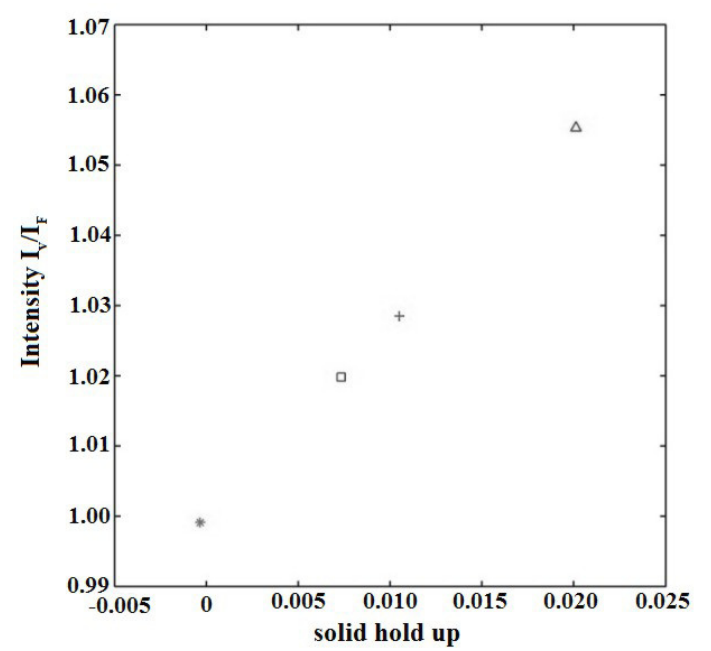

Fig. 3. Relative intensity $I_{\mathrm{V}} / I_{\mathrm{F}}$ against the solid hold up of four samples.

\subsection{DCCR experiments}

In the first stage of treatment, represented by the tests of fluid parameters, it was employed the methodology of experimental planning called Design Central Composite Rotatable (DCCR). The variables studied were gas velocity, gas flow, flow of solids, and the solid concentration in the riser. The test conditions were steady state and varying the velocity of both phases.

\subsection{Uncertainty evaluation}

By calculating the relative experimental standard uncertainty it can be shown a quite different behavior of equivalent solid flow stability for all concentrations, i.e., the highest concentration has the highest uncertainty as given in Figure 4: the means are depicted as a circle for empty

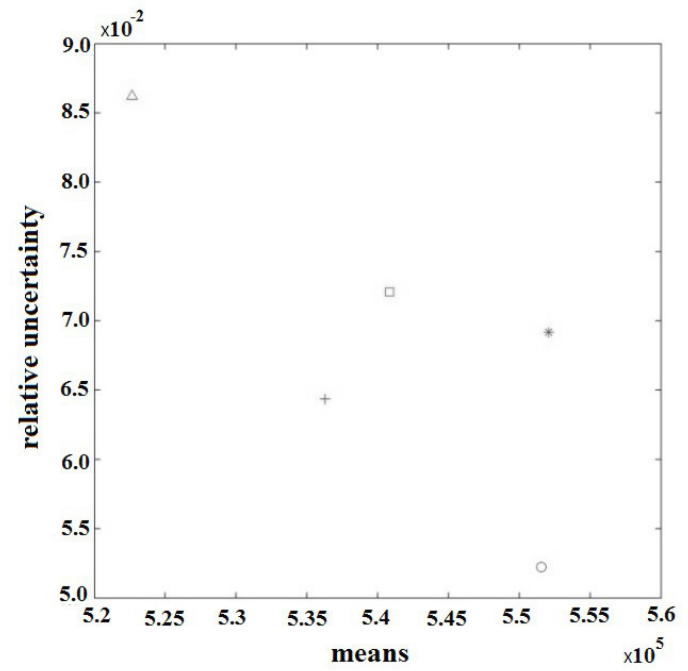

Fig. 4. Relative standard uncertainty versus the means of $I_{\mathrm{V}}$ in circle and of four catalyst concentrations.

tube and as a star for the lowest concentration, a cross and a square for intermediate concentrations while the highest concentration is depicted as a triangle.

Even that $\mathrm{o}$ and $*$ means are quite close, around $5.5 \times 10^{5}$; the lowest concentration in star induces some uncertainty in the transmission measurement. The triangle shows that the uncertainty is the highest for the lowest intensity due to the highest attenuation, their mean standing around $5.2 \times 10^{5}$, which is consistent with the monitoring graphs of the pressure by means of CFPEU control. According to what is stored in the database, as solid concentration increases the $\Delta p$ inside the riser will follow and oscillations in solid flow will also increase. As experiments show, the uncertainty in solid hold up measurement is a function of $\Delta p$ inside the riser.

To calculate the combined uncertainty in solid hold up by means of gamma-ray transmission measurements and equation (1), for the catalyst concentration whose mean's relative standard uncertainty is given in Figure 4. To calculate the combined standard uncertainty of hold up measured at riser diameter, equation (10) was used [16]

$$
\begin{aligned}
u_{c}^{2}(y) & =\sum_{i=1}^{N}\left[\frac{\partial f}{\partial x_{i}}\right]^{2} u^{2}\left(x_{i}\right) \\
u\left(y_{l}, y_{m}\right) & =\sum_{i=1}^{N} \sum_{j=1}^{N} \frac{\partial y_{l}}{\partial x_{i}} \frac{\partial y_{m}}{\partial x_{j}} u\left(x_{i}\right) u\left(x_{j}\right) r\left(x_{i}, x_{j}\right) .
\end{aligned}
$$

Correlation in radioactive measurements is considered in the literature [17], therefore, the use of equation (11), that takes into account the correlation between two variables, as input values to be included in equation (10). The correlation coefficient $r\left(x_{i}, x_{j}\right)$ gives values around 0.1 , for the measured intensities $I_{\mathrm{V}}$ and $I_{\mathrm{F}}$. A comparison with equation (10) was carried out and it shows that additional term in equation (11) is of a negligible contribution, in the seventh decimal place. 


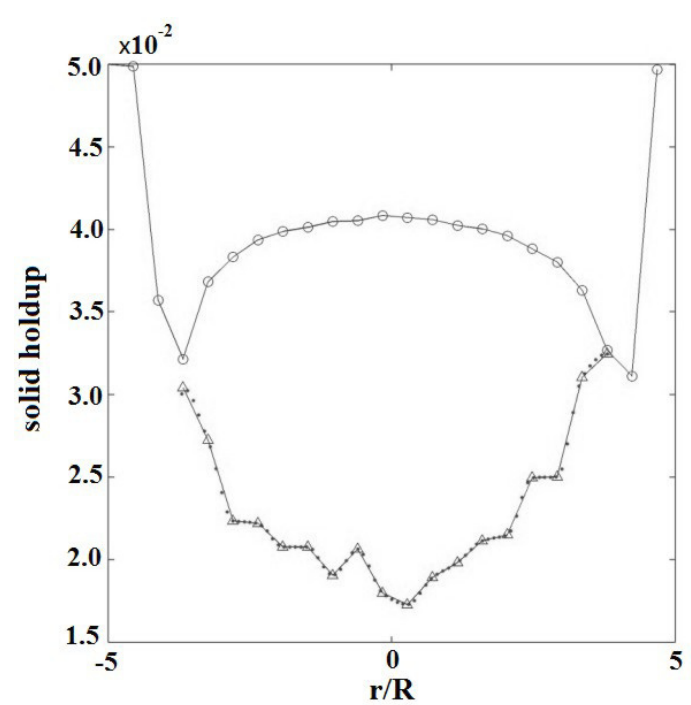

Fig. 5. Radial distribution of solid hold up depicted as triangles, interpolation points and tube-riser profile depicted as circles.

Calculating the uncertainty in data from the radial distribution of hold up the scanned scalars $I_{\mathrm{V}}$ and $I_{\mathrm{F}}$ are significantly correlated, the $r\left(x_{i}, x_{j}\right)$ gives values around 0.98. Calculation with equation (11), shows that its contribution in fourth decimal place should be taken into account.

\subsection{Mass solid flow}

Considering that gamma ray beam transmitted through an object can be approximated by a cylinder shape, with a volume given by beam diameter $\varphi$ and internal tube diameter $D$ :

$$
v=\pi\left(\frac{\varphi}{2}\right)^{2} D
$$

Measurement of density was carried out to evaluate an approximation for the volume $v$ by means of gamma-ray transmission by using an expression in which equation (9) is based:

$$
\rho_{m}=\frac{1}{\mu / \rho D} \ln \left(\frac{I_{\mathrm{V}}}{I_{\mathrm{F}}}\right)
$$

with $\mu / \rho$ as the linear attenuation coefficient and on the left hand side the mean density along gamma ray path length. During solid flow circulating the $\rho_{m}$ value corresponds to the catalyst density at a measuring point in the riser. Calculation with gamma-ray beam transmitted in equation (13) and the volume from equation (12) gives $m_{c}=v \rho_{m}$, the catalyst mass. An aluminum block was used for testing, as a comparison of $m_{c}$ against the mass measured in a scale, and they came close to only about $3 \%$ error.

The volume in equation (12) is associated to a volume $V$ given by

$$
V=\pi\left(\frac{D}{2}\right)^{2} \varphi
$$

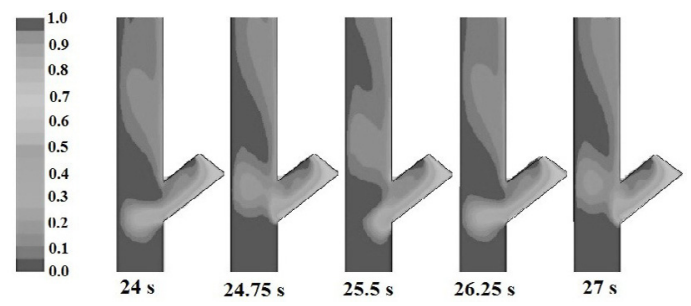

Fig. 6. Profiles of instantaneous volume fraction of solids in the entrance region at five different instants.

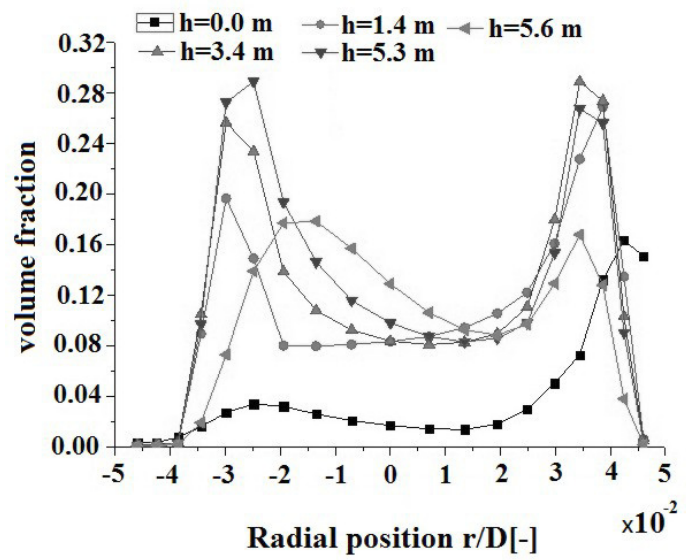

Fig. 7. Radial profiles of solid volume fraction at different heights of riser.

where the cross-section area of the tube-riser is multiplied by the beam diameter. Then, the corresponding catalyst mass becomes: $M_{c}=V \rho_{m}$.

\section{Results and discussions}

Radial distribution of solid hold up:

Figure 6 illustrates the profile of the catalyst at the riser inlet for different instants of operation. There is a kind of cycle for the entry of solids. Due to the inclination of duct that feeds the riser with catalyst, at a distance approaching the entrance of the riser, a certain amount of solids peels off the flow of catalyst by contacting air flow and being dragged to the same upper regions of the riser. Due to the collision with the catalyst particles air flow deviates slightly from its initial trajectory (parallel to the walls of the riser), creating a preferential path along the wall of the riser, opposite to the entrance of the catalyst. The airflow stabilizing try again when another quantity of catalyst detaches itself from the sliding bed, which flows through the supply duct, and again the interaction cycle of air and catalyst is restarted. As the air mixture over the riser ascends in radial distribution, it becomes more homogeneous - then it attains a region of fully developed flow.

Figure 7 illustrates the radial volume fraction profile at different axial points for catalyst through riser, respectively. According to distinguishing solid concentration, 


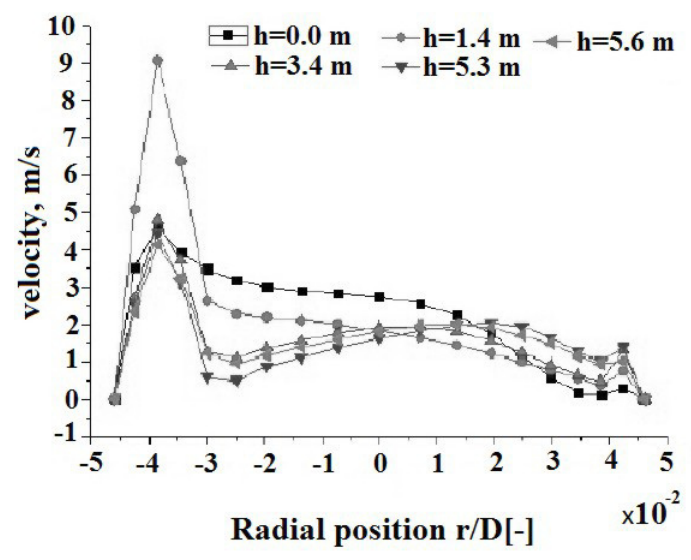

Fig. 8. Radial profiles of solid velocità at different heights of riser.

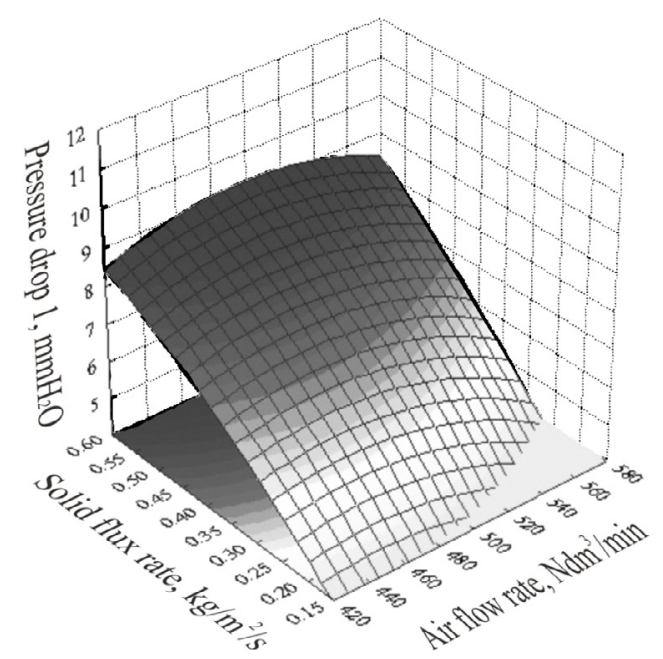

Fig. 9. Pressure drop at the bottom of the riser.

the riser can be divided into different parts: the bottom zone, the middle zone and the top zone. Due to vortex characteristic in the bottom zone, the solid concentration varies quickly with the change of the radial and axial positions. The length of the bottom zone was identified as about $1.4 \mathrm{~m}$. In the middle zone, the clusters mainly appear near the wall, which move downwards or upwards at the same time. In the dense top zone the particles assemble more homogeneous. Therefore, the solids concentration in the center region increases gradually along the riser when the flow is further developed.

Figure 8 shows radial profiles of solid velocities at different heights in the riser. Due to the inlet of catalyst, which forces the flow of compressed air to go preferably to the wall opposite to that entry, the velocity values close to this region are generally larger. Notice also that the entry region occurs in the vicinity of $1.4 \mathrm{~m}$, confirming the predictions made with the help of Figure 7.

To evaluate catalyst mass that flows in the riser by means of equations (12) and (13), the mass flow velocity is taken into account. A mass flow velocity measured at

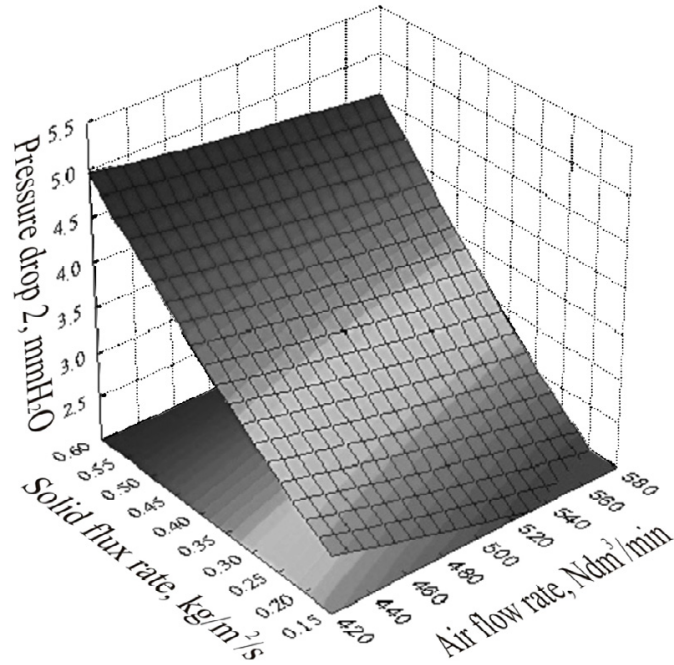

Fig. 10. Total pressure drop.

experimental conditions is $Q=2 \mathrm{~m} \mathrm{~s}^{-1}$, that corresponds to $\varphi / 2.75 \mathrm{~ms}$, which is beam diameter per millisecond. According to equation (14), $M_{c}=f(\varphi)$, an $M_{s}$ mass will be ascending in riser at $M_{c} / 2.75 \mathrm{~ms}$. Corresponding mass flow rate $W$ as a function of $M_{s}$, and of tube cross-section area was calculated as the usual expression: $W=\mathrm{kg} \mathrm{A}^{-1} \mathrm{~s}^{-1}, M_{s}=242.2 \mathrm{~g} \mathrm{~s}^{-1}$, or $M_{s}=7.266 \mathrm{~kg}$ for $30 \mathrm{~s}$, in counting time.

Acknowledgements. The authors are grateful to $\mathrm{CNPq}$ for scholarships and financial support, and to Dr. Waldir Martignoni of Petrobras for technical assistance.

\section{References}

1. T.M. Knowlton, Tools and techniques for diagnosing and solving operating problems in fluidized bed systems, Oil Gas Sci. Tech. 55, 209-217 (2000)

2. H. Arastoopour, D. Gisdaspow, Vertical pneumatic using four hydrodynamic models, Ind. Eng. Chem. 18, 123-130 (1979)

3. S.N. Khadzhiev, I.M. Gerzeliev, V.M. Kapustin, Kh.M. Kadiev, K.I. Dement'ev, O.A. Pakhmanova, Catalytic cracking in today's deep-conversion refinery, Petro. Chem. 51, 32-38 (2011)

4. V.A. dos Santos, C.C. Dantas, C.L. Luna-Finkler, J.E.G. de Souza, Principal component analysis in an experimental cold flow model of a fluid catalytic cracking unit by gammametry, Prog. Nucl. Energy 53, 1114-1118 (2011)

5. D.C. Montgomery, Design and Analysis of Experiments, 4th edn. (John Wiley \& Sons, 1997)

6. C. Derouin, D. Nevicato, M. Forissier, M.G. Wild, J. Bernard, Hydrodynamics of riser units and their impact on FCC operation, Ind. Eng. Chem. Res. 36, 4504-4511 (1997)

7. IAEA - International Atomic Energy Agency, Industrial Process Gamma Tomography (IAEA-TECDOC-1589, 2007) 
8. C.C. Dantas, R. Narain, V.A. dos Santos, A.C.B.A. de Melo, Catalyst concentration distribution in fluidized bed by gamma-ray absorption, J. Radioanal. Nucl. Chem. 269, $425(2006)$

9. C.C. Dantas, R. Narain, V.A. dos Santos, A.C.B.A. de Melo, R.V. Grieken, Precise gamma ray measurements of the radial distribution of a cracking catalyst at diluted concentrations in a glass riser, Nucl. Instrum. Methods Phys. Res. B, Beam Interact. Mater. Atoms 251, 201-208 (2006)

10. C.C. Dantas, S.B. Melo, E.F. Oliveira, F.B.P. Simões, M.G. dos Santos, V.A. Santos, Measurement of density distribution of a cracking catalyst in experimental riser with a sampling procedure for gamma ray tomography, Nucl. Instrum. Methods Phys. Res. B 266, 841-848 (2008)

11. G.V. Vasconcelos, S.B. Melo, C.C. Dantas, I. Malta, R. Oliveira, E.F. Oliveira, A particle system approach to industrial topographic reconstruction, Meas. Sci. Technol. 22, 104003-104010 (2011)
12. B.J. Harris, J.F. Davidson, Y. Xue, Axial and radial variations of flow in circulating fluidized bed risers, CFB Tech. IV, 103-110 (1994)

13. N. Mostoufi, J. Chaouki, Flow structure of solids in gassolid fluidized beds, Chem. Eng. Sci. 59, 4217-4227 (2004)

14. S. Nova, S. Krol, H. de Lasa, Particle velocity and particle clustering in down-flow reactors, Power Technol. 148, $172-185$ (2004)

15. Q.H. Wang, Q. Gao, H.S. Shi, X.C. Wu, Z.Y. Luo, K.F Cen, Formation, structure and motion of clusters in circulating fluidized bed, J. Zhejiang University (Eng. Sci.) 40, 118-122 (2006)

16. M.J. Rhodes, D. Geldart, Model for the CFB, Power Technol. 53, 155-162 (1987)

17. M. Horio, K. Morishita, O. Tachibana, N. Murata, Solid distribution and movement in CFBs, CFB Tech. II, 147-154 (1988) 